\title{
Gene expression analysis of potential genes and pathways involved in the pathogenic mechanisms of parvovirus B19 in human colorectal cancer
}

\author{
WEI-PING ZHANG ${ }^{1}, \mathrm{HUA} \mathrm{YANG}^{2}, \mathrm{HONG} \mathrm{CHEN}^{3}, \mathrm{HAI-RONG} \mathrm{ZHU}{ }^{4}, \mathrm{QUAN} \mathrm{LEI}^{4}, \mathrm{YUN}-\mathrm{HONG} \mathrm{SONG}^{2}$, \\ ZHONG-MING DAI ${ }^{1}$, JING-SHAN SUN ${ }^{1}$, LI-LI JIANG ${ }^{1}$ and ZHAN-GUO NIE ${ }^{1}$ \\ Departments of ${ }^{1}$ Gastroenterology and Hepatology, ${ }^{2}$ Outpatients, ${ }^{3}$ Blood Transfusion and ${ }^{4}$ Medical Administration, \\ Urumqi Military General Hospital, Urumqi, Xinjiang 830000, P.R. China
}

Received August 29, 2013; Accepted April 10, 2014

DOI: $10.3892 / \mathrm{ol} .2014 .2151$

\begin{abstract}
In order to investigate the pathogenic mechanisms of parvovirus B19 in human colorectal cancer, plasmids containing the VP1 or VP2 viral capsid proteins or the NS1 non-structural proteins of parvovirus B19 were constructed and transfected into primary human colorectal epithelial cells and LoVo cells. Differential gene expression was detected using a human genome expression array. Functional gene annotation analyses were performed using Database for Annotation, Visualization and Integrated Discovery v6.7 software. Gene ontology (GO) analyses revealed that VP1-related functions included the immune response, immune system process, defense response and the response to stimulus, while NS1-associated functions were found to include organelle fission, nuclear division, mitosis, the M-phase of the mitotic cell cycle, the mitotic cell cycle, M-phase, cell cycle phase, cell cycle process and cell division. Pathway expression analysis revealed that VP1-associated pathways included cell adhesion molecules, antigen processing and presentation, cytokines and the inflammatory response. Moreover, NS1-associated pathways included the cell cycle, pathways in cancer, colorectal cancer, the wnt signaling pathway and focal adhesion. Among the differential genes detected in the present study, 12 genes were found to participate in general cancer pathways and six genes were observed to participate in colorectal cancer pathways. NS1 is a key molecule in the pathogenic mechanism of parvovirus B19 in colorectal cancer. Several GO categories, pathways and genes were selected and may be the key targets through which parvovirus B19 participates in colorectal cancer pathogenesis.
\end{abstract}

Correspondence to: Dr Wei-Ping Zhang, Department of Gastroenterology and Hepatology, Urumqi Military General Hospital, 359 West Youhao Road, Urumqi, Xinjiang 830000, P.R. China

E-mail: weiping_zhang@hotmail.com

Key words: colorectal cancer, parvovirus B19, pathogenesis, microarray, human

\section{Introduction}

Colorectal cancer is the third most common type of cancer and the second most frequent cause of cancer mortality in numerous industrialized countries (1). The majority of tumors arise sporadically with no clear cause or genetic predisposition. Several risk factors have been considered as causes of colorectal cancer, but little has been confirmed. Viruses are among the few known causes of cancer and contribute to various malignancies worldwide (2). Previous studies on viral etiology in colon cancer have reported contradictory findings $(3,4)$.

Parvovirus B19 (B19) is a non-enveloped virus with a linear, single-stranded DNA genome. The B19 viral genome encodes three proteins: The non-structural protein, NS1, and two viral capsid proteins, VP1 and VP2 (5). In our previous study, significantly higher levels of B19 nucleic acids and proteins were found in neoplastic colon tissues (6). This finding indicates that an association may exist between B19 infection and the development of colon neoplasia.

Infection with parvovirus B19 is a global concern. The infection rate is similar in the United States, Europe and Asia, with $\sim 50 \%$ of 15 -year-old adolescents and $>60 \%$ of adults being seropositive (5). A previous study has shown that B19 infection may contribute to the pathogenesis of acute lymphoblastic and myeloblastic leukemia (7). However, few studies have investigated B19 in solid tumors or the mechanisms or regulatory proteins that could be involved. Therefore, it is important to establish whether B19 contributes to the pathogenesis of colorectal cancer and its underlying mechanism.

The present study aimed to investigate the pathogenic mechanisms underlying B19 in colon carcinoma by analyzing differential gene expression and biological functions, through assessing the changes in primary human colorectal epithelial cells (HCECs) and LoVo cells following transfection with plasmids containing VP1, VP2 and NS1.

\section{Materials and methods}

Plasmid construction. The recombinant eukaryotic cell inducible expression vectors, pReceiver-M03-VP1, 
pReceiver-M33-VP2 and pReceiver-M16-NS1, were constructed by inserting B19 full-length VP1, VP2 and NS1 complementary DNA into pReceiver-M03, pReceiver-M33 or pReceiver-M16 (GeneCopoeia, Inc., Rockville, MD, USA), respectively. The VP1, VP2 and NS1 sequences were amplified by polymerase chain reaction (PCR) analysis using the pGEM/1-B19 plasmid provided by Professor J.P. Clewley at the Central Public Health Laboratory (London, UK) as the template, which contained the B19 full-length open reading frame. The presence of the recombinant plasmid was confirmed using DNA sequencing.

Cell culture and transfection. Samples of normal human colon tissue $>10 \mathrm{~cm}$ distant to the tumors was obtained from patients with colorectal adenocarcinoma. HCECs were isolated from the normal human colorectal tissue and were washed several times using phosphate-buffered saline (PBS) containing penicillin, streptomycin and amphotericin B using thermolysin and collagenase type I (Sigma-Aldrich, St. Louis, MO, USA), as described previously (8). The cells were then cultured in Epithelial Cell Growth Medium-2 (ScienCell Research Laboratories, Carlsbad, CA, UDA) containing amphotericin B. LoVo carcinoma cells were obtained from the American Type Culture Collection (Rockville, MD, USA) and were cultured in Dulbecco's modified Eagle's medium containing $10 \%$ fetal bovine serum. All cells were cultured at $37^{\circ} \mathrm{C}$ in an atmosphere containing $5 \% \mathrm{CO}_{2}$. All procedures were performed in accordance with standard guidelines for the study of humans and were approved by the Research Ethics Committee of Urumqi Military General Hospital (Urumqi, China). All patients provided written informed consent.

The cells were transfected with pReceiver-M03-VP1, pReceiver-M33-VP2 and pReceiver-M16-NS1, using pReceiver-M03,pReceiver-M33 and pReceiver-M16 as controls. Transfection was performed using Lipofectamine ${ }^{\circledR}$ LTX and PLUS $^{\text {тм }}$ Reagents (Invitrogen Life Technologies, Carlsbad, CA, USA) according to the manufacturer's instructions, with untransfected cells used as blank controls.

Fluorescence microscopy. The expression of enhanced green fluorescent protein (eGFP), enhanced cyan fluorescent protein (eCFP) and enhanced yellow fluorescent protein (eYFP) in the transfected HCECs and LoVo cells was observed using a fluorescence microscope (TE2000-U, Nikon Corporation, Tokyo, Japan) equipped with a fluorescence filter. Digital images of the cells were captured using a spot camera system (Nikon Corporation).

Flow cytometric analysis. The cells $\left(1 \times 10^{6}\right)$ were fixed in $75 \%$ alcohol for $12-16 \mathrm{~h}$ at $4^{\circ} \mathrm{C}$, followed by ethidium bromide $(50 \mu \mathrm{g} / \mathrm{ml})$ and RNase $(100 \mu \mathrm{g} / \mathrm{ml})$ treatment at $25^{\circ} \mathrm{C}$ for $30 \mathrm{~min}$. Analysis was performed using a flow cytometer (FACScan; Becton Dickinson, Bedford, MA, USA).

Reverse transcription PCR (RT-PCR) analysis. RNA was extracted $24 \mathrm{~h}$ and $48 \mathrm{~h}$ subsequent to transfection, and RT-PCR was performed. The following primer pairs were used: Vp1 forward, ttctgcatgactgctactgga and reverse, atc ccctagaaaacccatcct; Vp2 forward, tatttgaggaggtggetgatg and reverse, ccaataaaggaacccagcaat; Ns1 forward, ggtggtctggga tgaaggtat and reverse, gtgttcccgcttacaacaaaa; and glyceraldehyde-3-phosphate dehydrogenase (GAPDH). forward, tcggagtcaacggatttggtcgta and reverse, tggcatggactgtggtcatgagtc.

Western blot analysis. Protein extraction was performed by washing the cells twice with ice-cold PBS, followed by homogenization in lysis buffer [50 mM HEPES (pH 7.5), $150 \mathrm{mM}$ $\mathrm{NaCl}, 10 \%$ glycerol, $1 \%$ Triton X-100, $1.5 \mathrm{mM} \mathrm{MgCl}_{2}, 1 \mathrm{mM}$ EDTA, $10 \mathrm{mM} \mathrm{Na}\left(\mathrm{PO}_{4}\right)_{2}, 25 \mu \mathrm{g} / \mathrm{ml}$ aprotinin and $25 \mu \mathrm{g} / \mathrm{ml}$ leupeptin] at $24 \mathrm{~h}, 48 \mathrm{~h}$ and $72 \mathrm{~h}$ post-transfection. The insoluble fraction was removed using centrifugation at $1,000 \mathrm{x} g$ for $15 \mathrm{~min}$ at $4^{\circ} \mathrm{C}$. Proteins were analyzed using electrophoresis (50 $\mu \mathrm{g}$ per lane) on $10 \%$ polyacrylamide gels and transferred to polyvinylidene fluoride (PVDF) membranes. Mouse monoclonal antibodies against the B19 proteins VP1 and VP2 (clone, R92F6; Chemicon, Billerica, MA, USA) and anti-NS1 antibodies [a gift from Professor Susanne Modrow (9) and Dr Simon Bredl, Institute of Medical Microbiology, University of Regensburg, Germany] were used to identify the proteins on the PVDF membranes. Horseradish peroxidase-conjugated goat antimouse secondary antibodies (Sigma-Aldrich) were detected using enhanced chemiluminescence western blot analysis reagents (Pierce Biotechnology, Inc., Rockford, IL, USA).

Microarray hybridization and data analysis. Microarray hybridization was performed by Shanghai Biochip Co., Ltd., (Shanghai, China) using an Agilent SurePrint G3 Human GE 8x60k microarray (Agilent Technologies, Santa Clara, CA, USA) that targeted 27,958 Entrez Gene RNAs and 7,419 long non-coding RNAs (reference). In brief, total RNAs from the transfected cells were extracted and purified using the Qiagen RNeasy ${ }^{\circledR}$ Mini kit (Qiagen, Hilden, Germany). Total RNA was amplified using the Low Input Quick Amp Labeling kit, One-Color (Agilent Technologies). For hybridization, each slide was hybridized with $1.65 \mu \mathrm{g}$ Cy-3 labeled complementary RNA using the Gene Expression Hybridization kit (Agilent Technologies) in a Hybridization Oven (Agilent Technologies) according to the manufacturer's instructions. Subsequent to $17 \mathrm{~h}$ of hybridization, the slides were washed in staining dishes (Thermo Fisher Scientific, Waltham, MA, USA) with Gene Expression Wash Buffer (Agilent Technologies) according to the manufacturer's instructions. The slides were scanned at a 3- $\mu \mathrm{m}$ resolution using the green dye channel in an Agilent Microarray Scanner (Agilent Technologies). The data were read using Feature Extraction Software 10.7 (Agilent Technologies), and were normalized using Quantile Algorithm, Gene Spring 11.0 software (Agilent Technologies).

The data from three replicates were averaged. Genes were defined as differentially expressed if the intensity ratio (Cy5) was found to increase or decrease $>2$-fold and if the intensity ratio (Cy5) showed the same direction of change (upregulated or downregulated) in all three experimental repeats. Gene ontology (GO) and pathway analyses were performed using Database for Annotation, Visualization and Integrated Discovery v6.7 software $(10,11)$.

Quantitative (q)PCR analysis. qPCR analysis using $\mathrm{SYBR}^{\circledR}$ Green (Invitrogen Life Technologies) was performed in 
Table I. Number of differentially-expressed genes in HCECs and LoVo cells transfected with plasmids.

\begin{tabular}{|c|c|c|c|c|c|c|}
\hline \multirow[b]{2}{*}{ Cell } & \multicolumn{2}{|c|}{ VP1 } & \multicolumn{2}{|c|}{ VP2 } & \multicolumn{2}{|c|}{ NS1 } \\
\hline & Upregulated, n & Downregulated, $\mathrm{n}$ & Upregulated, n & Downregulated, n & Upregulated, n & Downregulated, $\mathrm{n}$ \\
\hline HCEC & 740 & 361 & 131 & 124 & 447 & 690 \\
\hline LoVo & 613 & 328 & 546 & 562 & 508 & 652 \\
\hline
\end{tabular}

HCECs, human colorectal epithelial cells.


Figure 1. Expression of VP1, VP2 and NS1 in HCEC and LoVo cells. (A-C) HCECs and (D-F) LoVo cells marked with green, cyan and yellow fluorescence (magnification, $\mathrm{x} 400$ ). (G) Reverse transcription polymerase chain reaction (RT-PCR) analysis revealing VP1, VP2 and NS1 mRNA expression in the HCECs and LoVo cells. (H) Western blot analysis showing VP1, VP2 and NS1 protein expression in the HCECs and LoVo cells. HCECs, human colorectal epithelial cells.

order to verify the results of the microarray analysis. Total RNA was extracted from the transfected cells. The RNA was reverse transcribed using Murine Leukemia Virus reverse transcriptase (Promega Corp., Madison, WI, USA). The expression of the 12 genes that were identified as being associated with apoptosis in the microarray analysis was determined using qPCR analysis with SYBR-Green I (Invitrogen Life Technologies). GAPDH was used as an internal control and distilled water was used as a negative control. The amplification reaction consisted of 10X PCR buffer, 1.25 units of JumpStart ${ }^{\mathrm{TM}}$ Taq (Sigma-Aldrich),


$100 \mathrm{ng}$ template and 0.2X SYBR-Green I (Amresco Inc., Solon, OH, USA) in a final volume of $50 \mu \mathrm{l}$. The reactions were performed using the StepOne ${ }^{\mathrm{TM}}$ Real-Time PCR System
(Applied Biosystems, Inc., Foster City, CA, USA). The mRNA expression of the 12 genes was normalized with GAPDH using the $2^{-\Delta \Delta C t}$ method (12). The primer sequences used for GAPDH and the 12 genes were retrieved from PrimerBank (http://pga.mgh.harvard.edu/primerbank/).

\section{Results}

VP1, VP2 and NS1 expression in HCECs and LoVo cells. Primary normal HCECs were isolated, cultured and transiently transfected with the pReceiver-M03-VP1, pReceiver-M33-VP2 and pReceiver-M16-NS1 constructs. The expression of eGFP-VP1, eCFP-VP2 and eYFP-NS1 in the HCECs and LoVo cells was analyzed and confirmed using fluorescence microscopy, RT-PCR analysis and western blot analysis. 


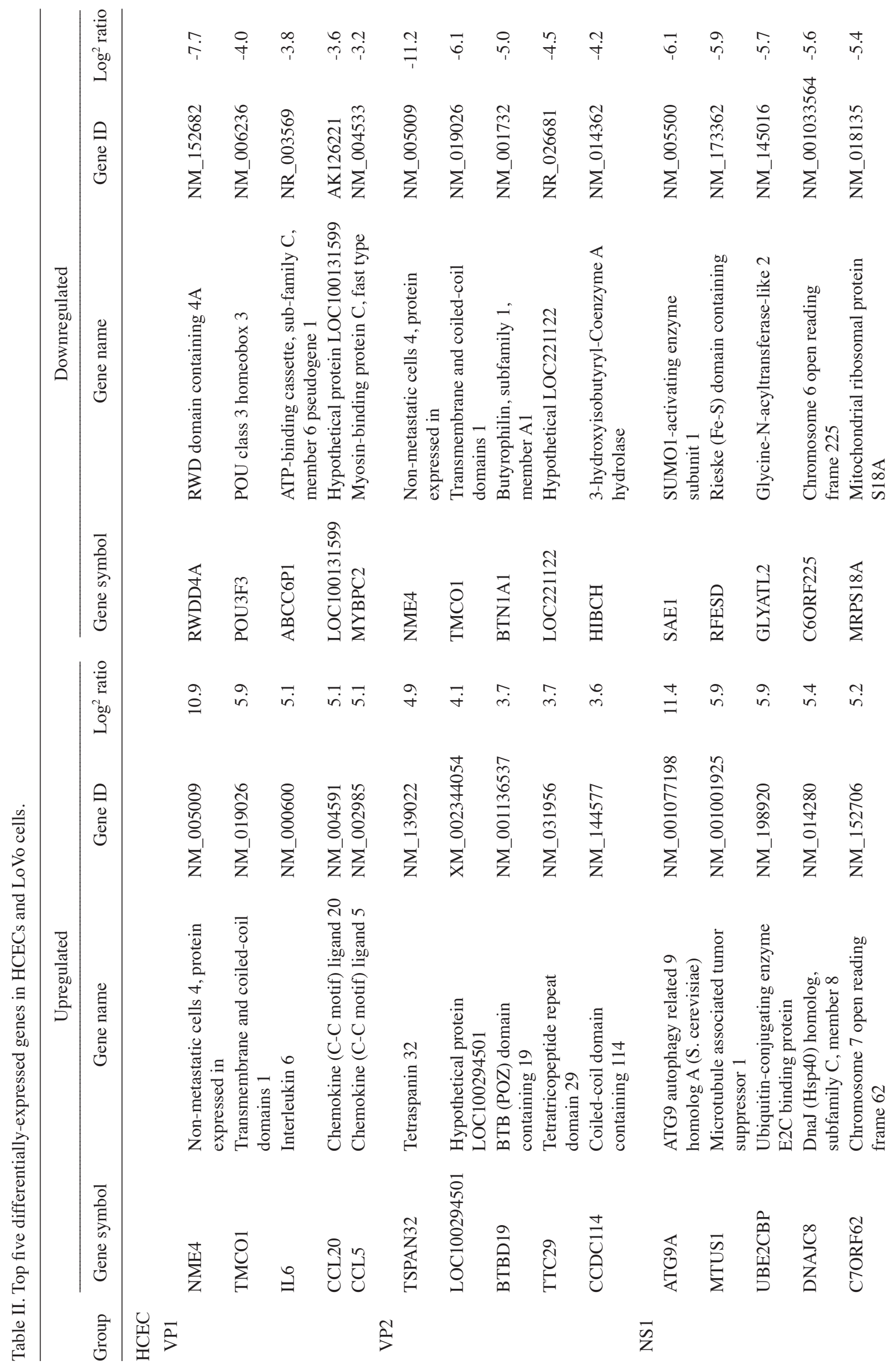




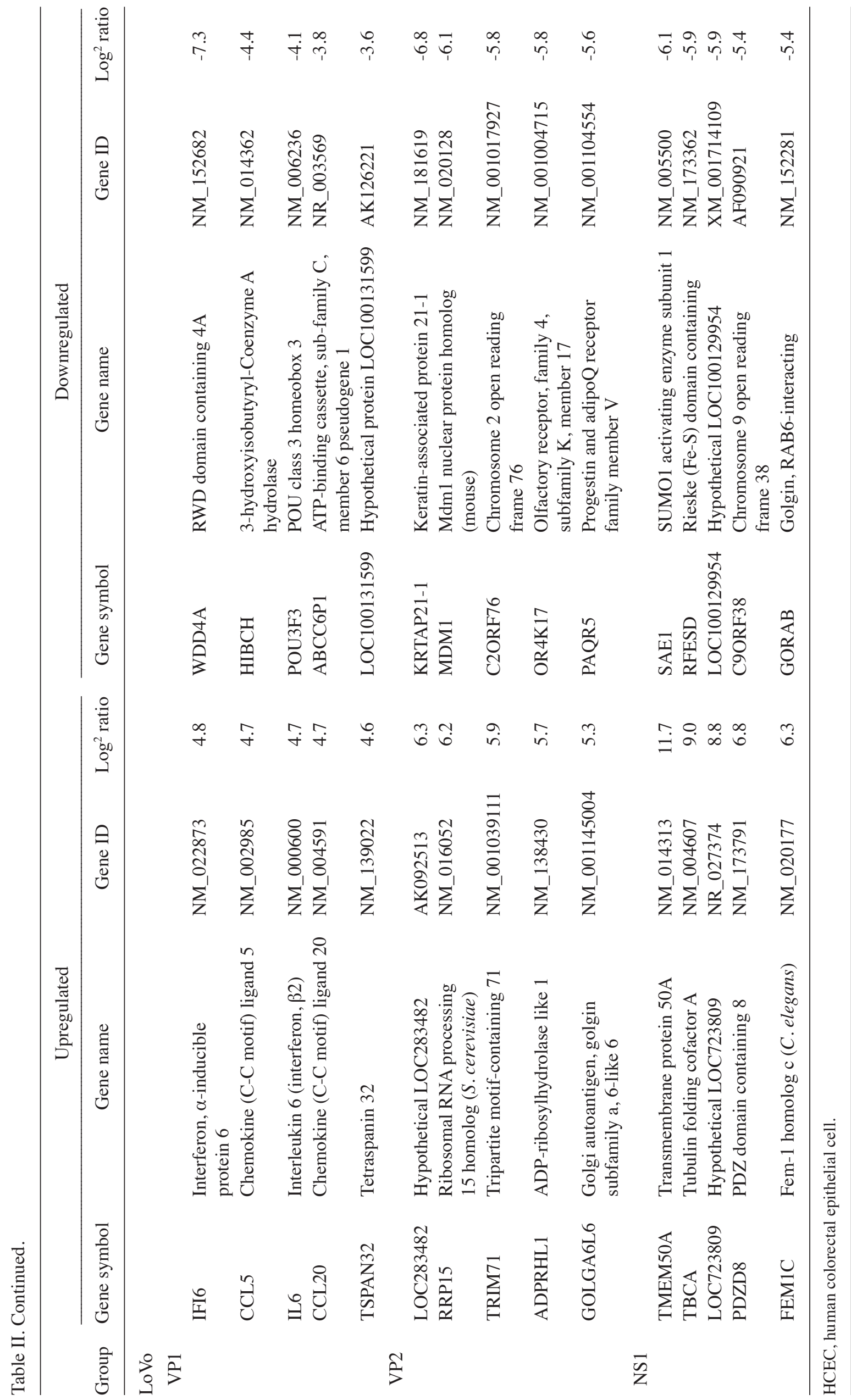


Table III. Fold-change of differentially-expressed genes associated with colorectal cancer.

\begin{tabular}{|c|c|c|c|c|c|c|c|}
\hline \multirow[b]{2}{*}{ Gene symbol } & \multirow[b]{2}{*}{ Genbank accession } & \multicolumn{3}{|c|}{ HCECs } & \multicolumn{3}{|c|}{ LoVo cells } \\
\hline & & VP1 & VP2 & NS1 & VP1 & VP2 & NS1 \\
\hline RAC3 & NM_005052 & - & - & 1.44 & - & - & 1.59 \\
\hline MYCL1 & NM_005376 & - & - & 1.29 & - & - & 1.06 \\
\hline APCDD1 & NM_153000 & - & 2.94 & - & 2.91 & - & - \\
\hline APC & NM_001127511 & - & - & 1.41 & - & - & -2.38 \\
\hline TCF7L2 & NM_030756 & - & - & - & - & 1.38 & 3.43 \\
\hline VTI1A & NM_145206 & - & - & -1.17 & - & - & -1.23 \\
\hline TP53INP2 & NM_021202 & 1.47 & - & - & - & - & - \\
\hline TP53I11 & NM_001076787 & - & - & -3.42 & - & - & -1.34 \\
\hline TP53INP1 & NM_033285 & - & - & - & 1.08 & - & - \\
\hline CRCX7 & NM_020311 & 1.77 & - & - & 1.63 & - & - \\
\hline BAX & NM_004324 & - & - & 1.23 & - & - & 1.05 \\
\hline TMBIM1 & NM_022152 & - & - & - & 1.61 & - & - \\
\hline LRP11 & NM_032832 & - & - & - & - & 3.04 & - \\
\hline VEGFA & NM_001025370 & - & - & 1.19 & - & - & - \\
\hline CCND1 & NM_053056 & - & - & 2.59 & - & - & - \\
\hline FOS & NM_005252 & - & - & -1.82 & - & - & -1.83 \\
\hline FOSB & NM_006732 & 1.66 & - & - & 1.27 & - & - \\
\hline FOSL1 & NM_005438 & 2.21 & - & - & 1.98 & - & 2.27 \\
\hline ID1 & NM_002165 & 1.24 & - & - & - & - & - \\
\hline FZD4 & NM_012193 & 3.40 & - & - & 2.85 & - & - \\
\hline FZD1 & NM_003505 & - & - & -1.14 & - & - & -1.11 \\
\hline FZD10 & NM_007197 & 1.15 & - & - & - & - & - \\
\hline
\end{tabular}

-, no significant difference $\left(\log ^{2}\right.$ ratio $>1$ or $\left.<-1\right)$; HCECs, human colorectal epithelial cells.

Marked green, cyan and yellow fluorescence, indicating the expression of VP1, VP2 and NS1, respectively, was observed at $24 \mathrm{~h}$ post-transfection, with the strongest expression observed after $48 \mathrm{~h}$ (Fig. 1A-F). The mRNA expression of VP1, VP2 and NS1 was detected using RT-PCR analysis in the transfected cells (Fig. 1G). To further assess the expression of eGFP-VP1, eCFP-VP2 and eYFP-NS1 in the HCECs and LoVo cells, the protein expression of VP1, VP2 and NS1 was confirmed using anti-VP1, -VP2 and -NS1 antibodies in western blot analysis (Fig. 1H).

Flow cytometric analysis. No significant changes in the cell cycle or apoptosis were identified in the HCECs transfected with pReceiver-M03-VP1, pReceiver-M33-VP2 or pReceiver-M16-NS1 and pReceiver-M03, pReceiver-M33 or pReceiver-M16. Similarly, no significant changes in cell cycle or apoptosis were identified in the LoVo cells transfected with pReceiver-M03-VP1, pReceiver-M33-VP2 or pReceiver-M16-NS1 and pReceiver-M03, pReceiver-M33 or pReceiver-M16.

Differential gene analysis. Using the human genome expression microarray, differential gene expression was detected in the HCECs and LoVo cells transfected with pReceiver-M03-VP1, pReceiver-M33-VP2 or pReceiver-M16-NS1 compared with those transfected with pReceiver-M03, pReceiver-M33 or
pReceiver-M16, respectively. The number of upregulated and downregulated genes $(\mathrm{P}<0.05$; false discovery rate $<0.05$; fold-change $>2.0$ ) are shown in Table I. The top five differential genes in the six groups are shown in Table II. The fold change of the differentially-expressed genes associated with colorectal cancer are shown in Table III.

GO analysis. The differentially-expressed genes were classified into different functional categories based on $\mathrm{GO}$ analysis for biological process, molecular function and cellular components. The primary GO categories for the upregulated genes in the HCECs and LoVo cells transfected with pReceiver-M03-VP1 included immune response, immune system process, defense response and response to stimulus, and for the downregulated genes was primarily cellular amino acid metabolic process. The predominant GO categories for the upregulated genes in the HCECs and LoVo cells transfected with pReceiver-M16-NS1 included organelle fission, nuclear division, mitosis, the M-phase of the mitotic cell cycle, the mitotic cell cycle, M-phase, cell cycle phase, cell cycle process and cell division (Table IV).

Pathway analysis. Significant pathways for the upregulated and downregulated differentially-expressed genes are shown in Table V. No pathways or specified pathways were found among the upregulated genes in the HCECs following 
Table IV. GO terms for the differentially-expressed genes.

Group

GO term

Genes, Genes,

Fold

Upregulation

VP1 in HCEC GO:0006955 immune response

GO:0002376 immune system process

GO:0006952 defense response

GO:0050896 response to stimulus

$\mathrm{n}$

$\%$ enrichment P-value FDR

inCEC No significant enrichment

NS1 in HCEC GO:0048285 organelle fission

GO:0000280 nuclear division

GO:0007067 mitosis

GO:0000087 M-phase of mitotic cell cycle

GO:0000278 mitotic cell cycle

GO:0000279 M-phase

GO:0022403 cell cycle phase

GO:0022402 cell cycle process

GO:0051301 cell division

VP1 in LoVo GO:0006955 immune response

GO:0002376 immune system process

GO:0050896 response to stimulus

VP2 in LoVo No significant enrichment

NS1 in LoVo GO:0048285 organelle fission

GO:0007067 mitosis

GO:0000280 nuclear division

GO:0000087 M-phase of mitotic cell cycle

GO:0000279 M-phase

GO:0000278 mitotic cell cycle

GO:0022403 cell cycle phase

GO:0022402 cell cycle process

GO:0051301 cell division

\begin{tabular}{|c|c|c|c|c|}
\hline 87 & 11.85 & 3.48 & $9.34 \mathrm{E}-25$ & 0.000 \\
\hline 105 & 14.31 & 2.90 & $1.06 \mathrm{E}-23$ & 0.000 \\
\hline 64 & 8.72 & 2.87 & $5.31 \mathrm{E}-14$ & 0.000 \\
\hline 197 & 26.84 & 1.55 & $3.60 \mathrm{E}-12$ & 0.000 \\
\hline - & - & - & - & - \\
\hline 20 & 4.49 & 4.33 & $1.90 \mathrm{E}-07$ & 0.000 \\
\hline 19 & 4.27 & 4.28 & $4.96 \mathrm{E}-07$ & 0.000 \\
\hline 19 & 4.27 & 4.28 & $4.96 \mathrm{E}-07$ & 0.000 \\
\hline 19 & 4.27 & 4.20 & $6.43 \mathrm{E}-07$ & 0.001 \\
\hline 24 & 5.39 & 3.21 & $1.72 \mathrm{E}-06$ & 0.002 \\
\hline 22 & 4.94 & 3.31 & 3.23E-06 & 0.005 \\
\hline 24 & 5.39 & 2.87 & $1.11 \mathrm{E}-05$ & 0.019 \\
\hline 28 & 6.29 & 2.45 & 2.87E-05 & 0.049 \\
\hline 18 & 4.04 & 3.02 & $1.02 \mathrm{E}-04$ & 0.175 \\
\hline 59 & 9.67 & 2.82 & $1.16 \mathrm{E}-12$ & 0.000 \\
\hline 72 & 11.80 & 2.38 & 8.37E-12 & 0.000 \\
\hline 149 & 24.43 & 1.40 & $2.42 \mathrm{E}-06$ & 0.004 \\
\hline- & - & - & - & - \\
\hline 22 & 4.35 & 4.36 & 3.38E-08 & 0.000 \\
\hline 21 & 4.15 & 4.33 & 8.32E-08 & 0.000 \\
\hline 21 & 4.15 & 4.33 & 8.32E-08 & 0.000 \\
\hline 21 & 4.15 & 4.26 & $1.12 \mathrm{E}-07$ & 0.000 \\
\hline 25 & 4.94 & 3.45 & $2.75 \mathrm{E}-07$ & 0.000 \\
\hline 25 & 4.94 & 3.07 & $2.26 \mathrm{E}-06$ & 0.004 \\
\hline 26 & 5.14 & 2.85 & 4.98E-06 & 0.009 \\
\hline 30 & 5.93 & 2.41 & $1.99 \mathrm{E}-05$ & 0.034 \\
\hline 18 & 3.56 & 2.77 & 2.89E-04 & 0.492 \\
\hline 16 & 4.43 & 4.78 & $1.28 \mathrm{E}-06$ & 0.002 \\
\hline 17 & 4.71 & 4.42 & $1.57 \mathrm{E}-06$ & 0.002 \\
\hline 18 & 4.99 & 3.99 & $2.78 \mathrm{E}-06$ & 0.004 \\
\hline 20 & 5.54 & 3.38 & 7.38E-06 & 0.012 \\
\hline 24 & 6.65 & 2.92 & $7.81 \mathrm{E}-06$ & 0.013 \\
\hline 24 & 6.65 & 2.92 & $7.81 \mathrm{E}-06$ & 0.013 \\
\hline- & - & - & - & - \\
\hline 175 & 25.36 & 1.32 & $1.35 \mathrm{E}-05$ & 0.020 \\
\hline 171 & 24.78 & 1.32 & $1.75 \mathrm{E}-05$ & 0.026 \\
\hline 171 & 24.78 & 1.31 & $3.08 \mathrm{E}-05$ & 0.046 \\
\hline 14 & 4.27 & 4.58 & 1.17E-05 & 0.019 \\
\hline 6 & 1.83 & 17.06 & $2.19 \mathrm{E}-05$ & 0.036 \\
\hline 11 & 3.35 & 5.24 & 4.67E-05 & 0.078 \\
\hline 11 & 3.35 & 5.24 & 4.67E-05 & 0.077 \\
\hline 7 & 2.13 & 10.14 & $5.98 \mathrm{E}-05$ & 0.099 \\
\hline- & - & - & - & - \\
\hline - & - & _ & - & - \\
\hline
\end{tabular}

Downregulation

VP1 in HCEC GO:0006520 cellular amino acid metabolic process

GO:0048037 cofactor binding

GO:0044106 cellular amine metabolic process

GO:0009308 amine metabolic process

GO:0019752 carboxylic acid metabolic process

GO:0043436 oxoacid metabolic process

VP2 in HCEC No significant enrichment

NS1 in HCEC GO:0043167 ion binding

GO:0046872 metal ion binding

GO:0043169 cation binding

VP1 in LoVo GO:0006520 cellular amino acid metabolic process

GO:0009069 serine family amino acid metabolic process

GO:0046394 carboxylic acid biosynthetic process

GO:0016053 organic acid biosynthetic process

GO:0008652 cellular amino acid biosynthetic process

VP2 in LoVo No significant enrichment

NS1 in LoVo No significant enrichment

HCEC, human colorectal epithelial cell; FDR, false discovery rate. 
Table V. Significant pathways for differentially-expressed genes.

\begin{tabular}{|c|c|c|c|c|c|c|}
\hline Group & Pathway name & $\begin{array}{c}\text { Genes, } \\
\mathrm{n}\end{array}$ & Genes & $\begin{array}{l}\text { Fold } \\
\text { change }\end{array}$ & P-value & FDR \\
\hline \multicolumn{7}{|l|}{ Upregulation } \\
\hline \multirow[t]{3}{*}{ VP1 in HCEC } & Cell adhesion molecules & 14 & $\begin{array}{l}\text { CD274, CD86, F11R, CDH1, ITGB8, ICAM1, } \\
\text { HLA-A, HLA-B, HLA-C, HLA-E, HLA-F, } \\
\text { HLA-G, HLA-DRB5, PTPRC, SDC4 }\end{array}$ & 2.4 & 0.005 & 5.9 \\
\hline & $\begin{array}{l}\text { Antigen processing and } \\
\text { presentation }\end{array}$ & 12 & $\begin{array}{l}\text { B2M, CTSS, HSP70B, HSP70B', HLA-A, } \\
\text { HLA-B, HLA-C, HLA-E, HLA-F, HLA-G, } \\
\text { HLA-DRB5, TAP1 }\end{array}$ & 2.7 & 0.010 & 1.1 \\
\hline & $\begin{array}{l}\text { Cytokines and } \\
\text { inflammatory response }\end{array}$ & 7 & CSF-2, CSF-3, IL1A, IL11, IL-6, IL-8, TNF & 4.6 & 0.002 & 3.2 \\
\hline VP2 in HCEC & None & & & & & \\
\hline \multirow[t]{4}{*}{ NS1 in HCEC } & Cell cycle & 21 & $\begin{array}{l}\text { NDC80, SPC25, BUB1, CDC20, CENPM, } \\
\text { CCNA2, CCND1, KIF20A, KIF23, MCM5, } \\
\text { PTTG1, PTTG2, PSMB8, TUBA1A, } \\
\text { TUBA4A, TUBB2C, TUBB, TUBB5, } \\
\text { TUBBP2, TUBBP1, UBE2E1 }\end{array}$ & 7.0 & 0.001 & 1.3 \\
\hline & Pathways in cancer & 9 & $\begin{array}{l}\text { BAX, APC, CCND1, FGF10, FGF17, } \\
\text { LAMA4, RAC3, VEGFA, WNT10B }\end{array}$ & 1.2 & 0.52 & 100 \\
\hline & Colorectal cancer & 4 & BAX, APC, RAC3, CCND1 & 2.0 & 0.32 & 99 \\
\hline & Focal adhesion & 6 & $\begin{array}{l}\text { MYLPF, CAV1, CCND1, LAMA4, RAC3, } \\
\text { VEGFA }\end{array}$ & 1.5 & 0.34 & 99 \\
\hline \multirow[t]{3}{*}{ VP1 in LoVo } & Cell adhesion molecules & 15 & $\begin{array}{l}\text { CD274, CD86, F11R, ITGB8, ICAM1, } \\
\text { HLA-A, HLA-B, HLA-C, HLA-E, HLA-F, } \\
\text { HLA-G, HLA-DRB5, SDC4, PVRL2, } \\
\text { PVRL3 }\end{array}$ & 3.2 & 0.000 & 0.2 \\
\hline & $\begin{array}{l}\text { Antigen processing } \\
\text { and presentation }\end{array}$ & 10 & $\begin{array}{l}\text { B2M, CTSS, HLA-A, HLA-B, HLA-C, } \\
\text { HLA-E, HLA-F, HLA-G, HLA-DRB5, TAP1 }\end{array}$ & 3 & 0.009 & 10 \\
\hline & $\begin{array}{l}\text { Cytokines and } \\
\text { inflammatory response }\end{array}$ & 4 & CSF-3, IL1A, IL-8, TNF & 2.9 & 0.015 & 84 \\
\hline VP2 in LoVo & None & & & & & \\
\hline NS1 in LoVo & Not significant & & & & & \\
\hline \multicolumn{7}{|l|}{ Downregulation } \\
\hline VP1 in HCEC & Not significant & & & & & \\
\hline VP2 in HCEC & None & & & & & \\
\hline NS1 in HCEC & Not significant & & & & & \\
\hline VP1 in LoVo & Not significant & & & & & \\
\hline VP2 in LoVo & Not significant & & & & & \\
\hline \multirow[t]{4}{*}{ NS1 in LoVo } & $\begin{array}{l}\text { Cytoskeletal regulation } \\
\text { by Rho GTPase }\end{array}$ & 16 & $\begin{array}{l}\text { ASPM, ENAH, IGFN1, MYLK, MYH13, } \\
\text { MYH6, PAK3, PAK2, RAC3, TTN, TUBB2A, } \\
\text { TUBB3, TUBB, TUBB5, TUBBP2, TUBBP1, }\end{array}$ & 2.2 & 0.013 & 13 \\
\hline & Wnt signaling pathway & 22 & $\begin{array}{l}\text { ARID1A, EP300, INO80, SMARCB1, APC, } \\
\text { ARRB2, DCHS1, FZD1, GNG3, MYH13, } \\
\text { MYH6, NFATC3, PPP3CB, PCDH18, } \\
\text { PCDH7, PCDHA5, PCDHGA5, PCDHGB7, } \\
\text { SVEP1, TTBK1, TCF7L2, MTCL1 }\end{array}$ & 1.2 & 0.25 & 96 \\
\hline & Pathways in cancer & 12 & $\begin{array}{l}\text { BAX, EP300, BMP4, FZD1, IGF1, PPARG, } \\
\text { PIAS4, RAC3, RUNX1T1, TCEB2, TCF7L2, } \\
\text { FOS }\end{array}$ & 0.78 & 0.91 & 100 \\
\hline & Colorectal cancer & 6 & BAX, APC, RAC3, FZD1, TCF7L2, FOS & 1.4 & 0.42 & 100 \\
\hline
\end{tabular}

P-value, enrichment of differentially-expressed genes; FDR, false discovery rate; HCEC, human colorectal epithelial cell. 
Table VI. Expression of 12 differentially-expressed genes detected using microarray analysis compared with qPCR analysis .

\begin{tabular}{lcrr}
\hline Gene symbol & Groups & qPCR & Microarray \\
\hline FOSB & VP1/HCEC & 1.6 & 1.7 \\
MYBPC2 & VP1/HCEC & -3.5 & -3.2 \\
APCDD1 & VP2/HCEC & 3.1 & 2.9 \\
NME4 & VP2/HCEC & -10.6 & -11.2 \\
RAC3 & NS1/HCEC & 1.7 & 1.4 \\
TP53I1 & NS1/HCEC & -4.0 & -3.4 \\
CRCX7 & VP1/LoVo & 1.5 & \\
ABCC6P1 & VP1/LoVo & -3.7 & -3.8 \\
LRP11 & VP2/LoVo & 2.9 & 3.0 \\
KRTAP21-1 & VP2/LoVo & -6.9 & -6.8 \\
TCF7L2 & NS1/LoVo & 3.2 & 3.4 \\
FOS & NS1/LoVo & -1.6 & -1.8 \\
\hline
\end{tabular}

Data are presented as the $\log ^{2}$ ratio. HCECs, human colorectal epithelial cells; qPCR, quantitative polymerase chain reaction.

transfection with pReceiver-M33-VP2 or in the LoVo cells following transfection with pReceiver-M33-VP2 or pReceiver-M16-NS1 (Table V). Similarly, no pathways were found among the downregulated genes in the HCECs following transfection with pReceiver-M03-VP1, pReceiver-M33-VP2 or pReceiver-M16-NS1, or in the LoVo cells following transfection with pReceiver-M03-VP1 or pReceiver-M33-VP2 compared with the cells transfected with the control plasmids.

Confirmation of microarray results using $q P C R$ analysis. To verify the microarray analysis data, the expression of the 12 differentially-expressed genes selected using microarray analysis was confirmed by qPCR analysis in the different groups. Consistent results were observed with regard to the nine genes in the microarray and qPCR analysis data (Table VI).

\section{Discussion}

Despite our current understanding of the genetic alterations associated with the progression of colon cancer, the specific etiology of colorectal cancer has yet to be elucidated. Epidemiological studies have indicated that environmental factors and host immunological characteristics may contribute to the initiation and progression of colon cancer. Infectious agents, primarily viral infection, are acquired through the environment and have the potential to alter numerous regulatory processes, which may result in the development of colorectal cancer. Our previous study showed that B19 infection may cause colon carcinoma (6). However, little is known regarding the pathogenic mechanisms responsible for B19-induced tumorigenesis.

B19 was discovered in 1974 and is the only Parvoviridae family member that is known to be pathogenic in humans. The genome of B19 has two large open reading frames encoding a single non-structural protein, NS1, and two capsid proteins, VP1 and VP2, which form an icosahedral capsid (5). The contribution of these viral proteins to B19 infectivity have yet to be experimentally demonstrated due to problems with in vitro culture and the lack of an infectious clone. Due to the difficulty in culturing B19 in vitro, little experimental evidence exists regarding the known and putative roles of B19 viral proteins in infectivity. In the present study, plasmids containing VP1, VP2 and NS1 were constructed and transfected into cultured HCECs and LoVo cells. Through the analysis of differentially-expressed genes and their functional enrichment, the present study aimed to identify potential targets to enable further investigation of the function of B19 in colon carcinoma, rather than to identify specific signaling pathways or molecules leading to colon carcinoma in which B19 participated.

Current understanding of the B19 viral proteins is primarily based on studies of other parvoviruses. The B19 NS protein is a multifunctional protein, for which sequence analysis has revealed that, in addition to transregulation of the p6 promoter $(13,14)$, NS contains motifs for nucleoside triphosphate (NTP) binding and hydrolysis (15) associated with helicase activity, thus indicating a role for the protein in B19 DNA replication. A previous study has also indicated that the NTP-binding motifs of NS play roles in the induction erythroid lineage cell apoptosis during B19 infection (16). VP2 is the major capsid protein, comprising $95 \%$ of the capsid and 58-kDa in size (17). Previous studies in insect cells have reported that VP2 can self-assemble into virus-like particles (17) and that it is capable of binding directly to blood group $\mathrm{P}$ antigen, which is the cellular receptor of B19 (18). VP1 is the minor capsid protein, which has an identical amino acid sequence to VP2, plus an extra 227 amino acids termed the VP1-unique region (VP1u)) at the amino terminus (19). Previous studies have shown that the VPlu, which is found on the exterior of the capsid, contains the primary neutralizing epitopes of B19 (20-22). Furthermore, a conserved phospholipase A2 motif has been identified in the VPlu of members of the Parvoviridae family, including B19 $(23,24)$. Two small $7.5-$ and $11-\mathrm{kDa}$ proteins, are encoded by the small abundant mRNA of B19 (25-27) and are unique among those parvoviruses that have so far been characterized. A number of proline-rich motifs are contained within the $11-\mathrm{kDa}$ protein and are conserved to the Src homology 3 binding domain of eukaryotic proteins (28); 
however, the function of the 7.5- and 11-kDa proteins in B19 replication and/or pathogenesis has yet to be elucidated.

In the present study, plasmids containing VP1, VP2 and NS1 were constructed for transfection into cultured HCECs and LoVo cells. Hundreds of differentially-expressed genes were identified in the HCECs and LoVo cells following VP1, VP2 and NS1 protein expression in different ontological pathways and functional GO groups. GO analyses revealed that the significant VP1-related ontology categories included that of immune response, immune system process, defense response and response to stimulus, while significant NS1-related ontology categories included organelle fission, nuclear division, mitosis, M-phase of the mitotic cell cycle, mitotic cell cycle, M-phase, cell cycle phase, cell cycle process and cell division. Pathway expression analysis identified that VP1-related pathways included cell adhesion molecules, antigen processing and presentation, cytokines and inflammatory response. Pathway expression analysis identified that NS1-related pathways included cell cycle, pathways in cancer, colorectal cancer, the wnt signaling pathway and focal adhesion. The functional GO categories and pathways associated with VP1 and NS1 that were identified in the present study were consistent with the functions of VP1 and NS1 reported previously $(6,9,13,14,16-23,28-30)$. This indicates that NS1 has a significant role in the pathogenesis of B19 in colorectal carcinoma.

In conclusion, the present study identified twelve differentially-expressed genes (BAX, EP300, BMP4, FZD1, IGF1, PPARG, PIAS4, RAC3, RUNX1T1, TCEB2, TCF7L2 and FOS) that were found to participate in general cancer pathways, and six genes (BAX, APC, RAC3, FZD1, TCF7L2 and FOS) that were found to specifically participate in colorectal cancer pathways. Furthermore, genes associated with cancer, including MYCL1, APCDD1, VTI1A, TP53INP2, TP53I11, TP53INP1, CRCX7, TMBIM1, LRP11, CCND1, FOSB, FOSL1, FZD4 and FZD10, were found to be differentially expressed. These may be the primary genes involved in regulating the pathogenesis of B19 in colorectal carcinoma. Moreover, NS1 may be the key molecule involved in the pathogenesis of B19 in colorectal carcinoma.

\section{Acknowledgements}

The present study was supported by grants from the National Natural Science Foundation of China (no. 30873472) and the China Postdoctoral Science Foundation (no. 20090461485).

\section{References}

1. Jemal A, Bray F, Center MM, et al: Global cancer statistics. CA Cancer J Clin 61: 69-90, 2011.

2. Pagano JS, Blaser M, Buendia MA, et al: Infectious agents and cancer: criteria for a causal relation. Semin Cancer Biol 14: 453-471, 2004.

3. Newcomb PA, Bush AC, Stoner GL, et al: No evidence of an association of JC virus and colon neoplasia. Cancer Epidemiol Biomarkers Prev 13: 662-666, 2004.

4. Ricciardiello L, Laghi L, Ramamirtham P, et al: JC virus DNA sequences are frequently present in the human upper and lower gastrointestinal tract. Gastroenterology 119: 1228-1235, 2000.

5. Young NS and Brown KE: Parvovirus B19. N Engl J Med 350: 586-597, 2004.

6. Li Y, Wang J, Zhu G, et al: Detection of parvovirus B19 nucleic acids and expression of viral VP1/VP2 antigen in human colon carcinoma. Am J Gastroenterol 102: 1489-1498, 2007.
7. Kerr JR, Barah F, Cunniffe VS, et al: Association of acute parvovirus B19 infection with new onset of acute lymphoblastic and myeloblastic leukaemia. J Clin Pathol 56: 873-875, 2003.

8. Perreault $\mathrm{N}$ and Beaulieu JF: Use of the dissociating enzyme thermolysin to generate viable human normal intestinal epithelial cell cultures. Exp Cell Res 224: 354-364, 1996.

9. von Poblotzki A, Gigler A, Lang B, Wolf H and Modrow S: Antibodies to parvovirus B19 NS-1 protein in infected individuals. J Gen Virol 76: 519-527, 1995.

10. Huang da W, Sherman BT and Lempicki RA: Bioinformatics enrichment tools: paths toward the comprehensive functional analysis of large gene lists. Nucleic Acids Res 37: 1-13, 2009.

11. Huang da W, Sherman BT and Lempicki RA: Systematic and integrative analysis of large gene lists using DAVID bioinformatics resources. Nat Protoc 4: 44-57, 2009.

12. Livak KJ and Schmittgen TD: Analysis of relative gene expression data using real-time quantitative PCR and the 2(-Delta Delta C(T)) method. Methods 25: 402-408, 2001.

13. Doerig C, Hirt B, Antonietti JP and Beard P: Nonstructural protein of parvoviruses B19 and minute virus of mice controls transcription. J Virol 64: 387-396, 1990.

14. Raab U, Beckenlehner K, Lowin T, et al: NS1 protein of parvovirus B19 interacts directly with DNA sequences of the p6 promoter and with the cellular transcription factors Sp1/Sp3. Virology 293: 86-93, 2002.

15. Momoeda M, Wong S, Kawase M, Young NS and Kajigaya S: A putative nucleoside triphosphate-binding domain in the nonstructural protein of B19 parvovirus is required for cytotoxicity. J Virol 68: 8443-8446, 1994.

16. Moffatt S, Yaegashi N, Tada K, Tanaka N and Sugamura K: Human parvovirus B19 nonstructural (NS1) protein induces apoptosis in erythroid lineage cells. J Virol 72: 3018-3028, 1998.

17. Kajigaya S, Fujii H, Field A, et al: Self-assembled B19 parvovirus capsids, produced in a baculovirus system, are antigenically and immunogenically similar to native virions. Proc Natl Acad Sci USA 88: 4646-4650, 1991.

18. Brown KE, Anderson SM and Young NS: Erythrocyte P antigen: cellular receptor for B19 parvovirus. Science 262: 114-117, 1993.

19. Ozawa K and Young N: Characterization of capsid and noncapsid proteins of B19 parvovirus propagated in human erythroid bone marrow cell cultures. J Virol 61: 2627-2630, 1987.

20. Saikawa T, Anderson S, Momoeda M, Kajigaya S and Young NS: Neutralizing linear epitopes of B19 parvovirus cluster in the VP1 unique and VP1-VP2 junction regions. J Virol 67: 3004-3009, 1993.

21. Rosenfeld SJ, Yoshimoto K, Kajigaya S, et al: Unique region of the minor capsid protein of human parvovirus B19 is exposed on the virion surface. J Clin Invest 89: 2023-2029, 1992.

22. Kawase M, Momoeda M, Young NS and Kajigaya S: Most of the VP1 unique region of B19 parvovirus is on the capsid surface. Virology 211: 359-366, 1995.

23. Lu J, Zhi N, Wong S and Brown KE: Activation of synoviocytes by the secreted phospholipase A2 motif in the VP1-unique region of parvovirus B19 minor capsid protein. J Infect Dis 193: 582-590, 2006.

24. Zádori Z, Szelei J, Lacoste MC, et al: A viral phospholipase A2 is required for parvovirus infectivity. Dev Cell 1: 291-302, 2001.

25. Luo W and Astell CR: A novel protein encoded by small RNAs of parvovirus B19. Virology 195: 448-455, 1993.

26. St Amand J and Astell CR: Identification and characterization of a family of $11-\mathrm{kDa}$ proteins encoded by the human parvovirus B19. Virology 192: 121-131, 1993.

27. St Amand J, Beard C, Humphries K and Astell CR: Analysis of splice junctions and in vitro and in vivo translation potential of the small, abundant B19 parvovirus RNAs. Virology 183: 133-142, 1991.

28. Fan MM, Tamburic L, Shippam-Brett C, Zagrodney DB and Astell CR: The small 11-kDa protein from B19 parvovirus binds growth factor receptor-binding protein 2 in vitro in a Src homology 3 domain/ligand-dependent manner. Virology 291: 285-291, 2001.

29. Moffatt S, Tanaka N, Tada K, et al: A cytotoxic nonstructural protein, NS1, of human parvovirus B19 induces activation of interleukin-6 gene expression. J Virol 70: 8485-8491, 1996.

30. Wan Z, Zhi N, Wong S, et al: Human parvovirus B19 causes cell cycle arrest of human erythroid progenitors via deregulation of the E2F family of transcription factors. J Clin Invest 120 : 3530-3544, 2010. 